\title{
Factores asociados a supervivencia en pacientes con tuberculosis en Lima, Perú
}

\author{
Antonio Bernabé-Ortiz
}

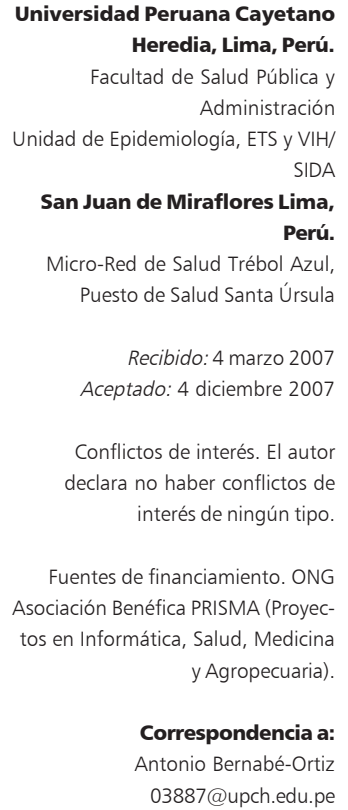

\section{Introducción}

A unque en Perú, la Estrategia Nacional para Control de la Tuberculosis ha tenido gran éxito en la reducción de la incidencia, morbilidad y mortalidad, todavía siguen dándose muertes secundarias a la enfermedad. ${ }^{1}$ Las mejoras en la detección de casos de TBC y en las tasas de cura aceleraron este proceso durante los años previos.

El análisis de los reportes de casos anuales de TBC mostró que la tasa de incidencia era constante antes de 1991. Los casos reportados aumentaron entre 1992 y 1993 como resultado de mejoras en la estandarización de la detección de $\operatorname{casos}^{2}$. Aunque los esfuerzos diagnósticos han seguido incrementándose desde 1993, la aparición de nuevos casos de TBC pulmonar ha declinado en todo el país, con un promedio de disminución mayor a 5,8\% por año (rango 1,9\% - 9,7\%). Esta tasa sugiere que aproximadamente $27 \%$ de los casos (158.000) y 70\% de las muertes (91.000) entre pacientes con expectoración positiva se evitaron entre 1991 y el 2000. Todo esto llevó a que el Perú saliera del grupo de países que la OMS considera responsable de la mayor parte de casos de TBC a nivel mundial ${ }^{3}$.

Asimismo, la combinación entre TBC y la infección por el VIH tienen una alta mortalidad; por lo que un diagnóstico y tratamiento oportuno son de suma importancia. ${ }^{4}$ Si bien en Perú, la epidemia del VIH es de tipo concentrada, la TBC es una enfermedad con alta prevalencia y una de las principales causas de muerte de personas infectadas con VIH y SIDA $^{5}$.

El propósito del presente estudio es evaluar la supervivencia en pacientes que inician tratamiento para TBC y determinar los factores pronósticos de la misma en una zona urbano-marginal de Lima.

\section{Material y Método}

Estudio observacional, longitudinal, retrospectivo, cuya población estuvo constituida por los pacientes con diagnóstico de TBC que recibieron tratamiento en la Micro-Red de Salud Trébol Azul (Pampas de San Juan de Miraflores, Lima), entre enero del 2000 y diciembre del 2005. Los datos, así como la fecha de defunción o alta, fueron tomados de los libros de seguimiento de Estrategia Nacional de Control de Tuberculosis y de las tarjetas de evolución clínica de cada paciente.

El tiempo de supervivencia estuvo definido como el número de días transcurridos desde el inicio de tratamiento anti-TBC hasta que ocurrió la muerte o la fecha de censura (final del tratamiento o pérdida al seguimiento).

Los datos obtenidos fueron digitados en una hoja de cálculo de Microsoft Excel versión 2000, para luego 
ser trasladadas a Epi-Info versión 3.3.2 y SPSS versión 11.0 (SPSS Inc., Chicago IL, E.U.A.) para el análisis. Se realizó la descripción de las características socio-demográficas, de antecedentes y clínicas. Cada variable fue valorada en forma estratificada para ser evaluada mediante el método de Kaplan-Meier como condición para cumplir el principio de riesgos proporcionales. Aquellas variables que estuvieron asociadas con supervivencia en el análisis bivariado fueron evaluadas individualmente a través del análisis de regresión de Cox para obtener el valor del Hazard Ratio (HR) y su respectivo intervalo de confianza. Luego, todas las variables con $\mathrm{p}<0,10$ fueron evaluadas en una serie de modelos multivariados para determinar la mejor combinación de factores independientes pronósticos de supervivencia.

\section{Resultados}

Durante el tiempo de estudio, se incluyeron 425 pacientes, con una proporción de varones de 59,1\% y un promedio de edad de 31,1 años (rango: 18-80). La mediana del tiempo de seguimiento fue de 178 días (RIQ: 170-212), con un total de 19 (4,5\%) muertes ocurridas y una tasa de mortalidad de 24,2 por 100.000 habitantes (95\% IC: 14,6-37,8).

Dentro de las características socio-demográficas, la edad y el grado de instrucción estuvieron asociados a supervivencia. Dentro de los antecedentes, únicamente la infección por el VIH estuvo asociada a mortalidad. Igualmente, dentro de las variables clínicas, sólo el índice de masa corporal (IMC) al inicio del tratamiento estuvo asociado a supervivencia (Tabla 1).

Al realizar el análisis de Kaplan-Meier entre el tiempo de supervivencia y cada una de las variables, la edad, el nivel de educación, la infección por el VIH, y el IMC $<18 \mathrm{~kg} / \mathrm{m}^{2}$ al inicio del tratamiento estuvieron asociados a supervivencia y fueron consideradas para el análisis final (Figura 1). A través del modelo de riesgos proporcionales de Cox, el modelo multivariado final mostró que el IMC $<18 \mathrm{~kg} / \mathrm{m}^{2}(\mathrm{p}=0,01)$, el grado de instrucción $(p=0,02)$ y la infección por $\operatorname{VIH}(p=0,03)$, fueron factores independientes asociados con supervivencia en pacientes con TBC (Tabla 2).

\section{Discusión}

Aunque existen varios estudios sobre supervivencia de TBC a nivel mundial ${ }^{6-8}$, muy pocos son los trabajos de Latinoamérica. Gran parte de este tipo de estudios presenta resultados de la asociación TBC e infección por VIH, siendo la mayoría de Brasil. En Perú,

\begin{tabular}{|c|c|c|c|c|}
\hline Variable & & $\begin{array}{l}\text { Fallecidos } \\
(\mathrm{n}=19)\end{array}$ & $\begin{array}{c}\text { Vivos } \\
(n=406)\end{array}$ & $\mathbf{p}^{*}$ \\
\hline \multicolumn{5}{|l|}{ Características socio-demográficas } \\
\hline Sexo & Masculino & $10(52,6 \%)$ & $241(59,4 \%)$ & 0,64 \\
\hline Edad & $\geq 30$ años & $15(78,9 \%)$ & $151(37,3 \%)$ & $<0,001$ \\
\hline Grado de instrucción & $\geq$ secundaria & $6(46,2 \%)$ & $153(75,7 \%)$ & 0,04 \\
\hline \multicolumn{5}{|l|}{ Antecedentes } \\
\hline Tratamiento previo & Positivo & $3(15,8 \%)$ & $67(16,5 \%)$ & 1,00 \\
\hline Infección por VIH & Positivo & $2(10,5 \%)$ & $3 \quad(0,7 \%)$ & 0,02 \\
\hline \multicolumn{5}{|l|}{ Características clínicas } \\
\hline Tipo de tuberculosis & Pulmonar & $14(73,7 \%)$ & $340(84,0 \%)$ & 0,22 \\
\hline Peso al inicio & $<50 \mathrm{~kg}$ & $5(27,8 \%)$ & $101(25,5 \%)$ & 0,79 \\
\hline IMC al inicio & $<18 \mathrm{~kg} / \mathrm{m}^{2}$ & $5(27,8 \%)$ & $28 \quad(7,2 \%)$ & 0,01 \\
\hline BK de espectoración al inicio & Positivo & $11(61,1 \%)$ & $288(72,7 \%)$ & 0,29 \\
\hline
\end{tabular}

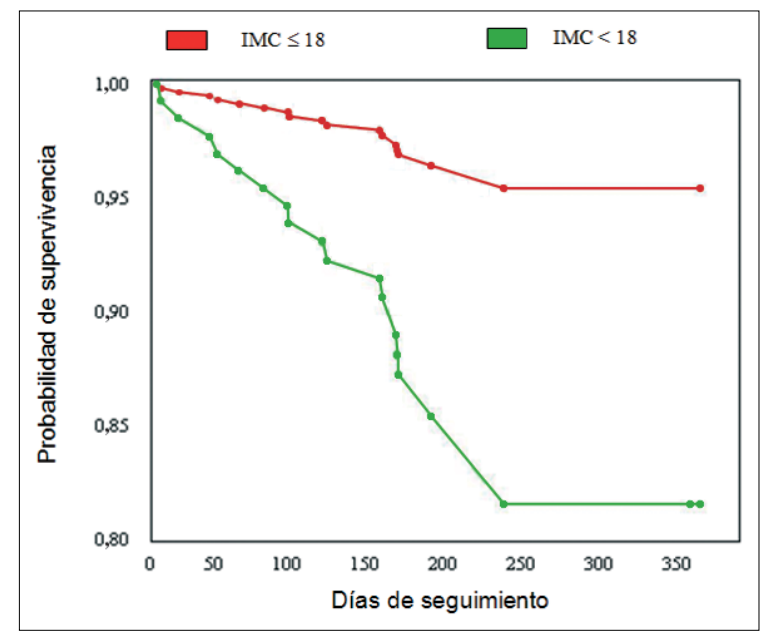

Figura 1. Curvas de KaplanMeier para supervivencia según el IMC al inicio del tratamiento. 
un trabajo previo publicado por Suárez y cols, demuestra que gracias a la estandarización de los métodos de diagnóstico y tratamiento del entonces Programa Nacional de Control, se logró evitar cerca de 91.000 muertes en un lapso de 10 años aproximadamente ${ }^{2}$.

En este estudio, el modelo multivariado final demuestra que la infección por el VIH es el principal factor que determina supervivencia, como ha quedado reportado en trabajos previos ${ }^{9,10}$. Aunque en Perú la epidemia de VIH es concentrada, siendo la prevalencia en estos pacientes con TBC de $1,2 \%$, es reconocido que los casos con la doble infección tienen una alta tasa de mortalidad con el subsecuente acortamiento del período de supervivencia. Sin embargo, todas las muertes ocurridas durante la terapia anti-TBC en este estudio han sido consideradas como muertes debidas a la enfermedad. Esto podría generar que los resultados varíen debido a los pocos casos de infección por VIH hallados, los cuales podrían haber fallecido por otras enfermedades oportunistas. No obstante, este estudio establece la misma relación entre TBC y VIH vista en previos estudios. De igual manera, un estudio retrospectivo previo realizado en el Hospital Cayetano Heredia en Lima, entre enero de 1986 y diciembre de 1993, mostró un tiempo de supervivencia para pacientes con la doble infección de 7,2 meses, comparado con 10,4 meses en pacientes que tenían sólo SIDA ${ }^{11}$.

Hasta la aparición del VIH/SIDA, la TBC era una enfermedad de buen pronóstico si es que se proveía el tratamiento en forma adecuada y oportuna. Antes de la quimioterapia, los análisis de supervivencia de pacientes con TBC pulmonar confirmada, diagnosticados entre 1925 y 1934 en una ciudad de Dinamarca, mostraron que la probabilidad de morir estaba entre 17 y 29\%, 32 y 43\%, y 42 y 55\% al año, 3 años y 5 años de seguimiento después del diagnóstico, respectivamen$\mathrm{te}^{12}$. Asimismo, en un estudio observacional de pacientes con diagnóstico de TBC pulmonar con expectoración positiva, entre 1928 y 1938, Thompson reportó que la probabilidad de morir en el primer año después del diagnóstico fue de $40 \%{ }^{13}$. Con la aplicación de quimioterapia anti-TBC efectiva, la tasa de mortalidad disminuyó hasta tal punto que una muerte por TBC, en cualquiera de sus formas, era considerada excepcional.

El IMC también estuvo asociado a mortalidad. Es reconocido que un valor de IMC por debajo del normal, incrementa el riesgo de morbilidad y mortalidad por cualquier enfermedad, especialmente por $\mathrm{TBC}^{14,15}$, así como a nivel poblacional la malnutrición se asocia a una mayor incidencia y severidad de la enfermedad visualizada a través de radiografías ${ }^{16,17}$. Un país en desarrollo, con altas tasas de malnutrición centrada en las zonas de alta pobreza, pone en riesgo a la comunidad, haciendo probable la diseminación de la enfermedad, con los nuevos riesgos que acarrea, tal como la multi-resistencia.

Este trabajo reconoce también que un mayor nivel de educación tiene un efecto protector para mortali$\operatorname{dad}^{1,5}$. Este efecto podría explicarse por un mayor conocimiento de la enfermedad, un rápido reconocimiento de los síntomas, por la necesidad y cumplimiento por parte del paciente de un tratamiento a largo plazo, así como por el reconocimiento temprano de las complicaciones. Asimismo, indirectamente una mejor educación puede asociarse a un mejor status socioeconómico, que podría reflejar una menor carencia de recursos, menor hacinamiento y mejores condiciones sanitarias.

La principal limitación de este estudio es que se utilizó el número de muertes ocurridas durante la terapia como marcador para mortalidad por TBC, por lo que estos resultados deben ser evaluados cautamente antes de ser generalizados. Sin embargo, este ha sido el criterio utilizado por la Estrategia para Control de la Tuberculosis para evaluar mortalidad por esta enfermedad en Perú. Otra limitación es que se trata de un estudio retrospectivo basado en reportes, lo cual limita la evaluación de otros reconocidos factores de riesgo como el nivel socio-económico ${ }^{16}$, la multi-resisten$\mathrm{cia}^{18}$, etc. A pesar de estas falencias, este estudio brinda la evaluación de la supervivencia en pacientes con tratamiento anti-TBC en una zona de alta prevalencia $^{19}$, como son las zonas urbano-marginales de Lima.

Los resultados del presente trabajo, aunque con cautela, sugieren que diversas intervenciones tienen el potencial de mejorar la supervivencia entre los pacientes infectados con TBC. Además de un pronto diagnóstico de las diversas formas de TBC a través de métodos diagnósticos estandarizados y del tratamiento apropiado y oportuno, y la continuidad del mismo ${ }^{20}$, los cuales son los puntos básicos de la estrategia DOTS, se deberían aplicar mejoras nutricionales en los pacientes, una mayor educación y capacitación a los trabajadores de salud y pacientes sobre la enfermedad y el control de la misma, así como un adecuado tamizaje y control de enfermedades concomitantes, especialmente la infección por el VIH que, aunque todavía es una epidemia concentrada en nuestro país, podría poner en riesgo nuestro sistema de salud pública.

Agradecimientos. El autor agradece a Julia Ríos, encargada del Programa de Control de Tuberculosis en la Micro-Red de Pampas de San Juan de Miraflores, por permitir el acceso a los datos del Programa. 


\section{Resumen}

Aunque en Perú, el Programa para Control de la Tuberculosis, ha tenido éxito en la reducción de la incidencia, morbilidad y mortalidad, todavía continúan dándose muertes secundarias a la enfermedad. Un estudio longitudinal retrospectivo se realizó en una zona urbano-marginal de Lima entre enero 2000 y diciembre de 2005. Se utilizó el análisis de Kaplan-Meier y regresión de Cox. Se incluyeron 425 pacientes con una tasa de mortalidad de 4,5\%. Los factores asociados a mortalidad fueron: índice de masa corporal (IMC) $<18 \mathrm{~kg} / \mathrm{m}^{2}$ al inicio del tratamiento, $\mathrm{HR}=4,89$ (95\% IC: 1,49-16,03) e infección por el VIH, HR = 5,78 (95\% IC: 1,11-29,99); mientras que el nivel de educación, HR = 0,28 (95\% IC: 0,10-0,83) estuvo asociado con supervivencia. En esta población que comienza tratamiento anti-TBC, el IMC y la infección por VIH estuvieron asociados con mayor riesgo de muerte; mientras que un mayor nivel educativo estuvo asociado con mejora en la supervivencia.

\section{Referencias}

1.- García-García M de L, Ponce-De-León A, García-Sancho M C, Ferreira-Reyes L, Palacios-Martínez M, Fuentes J, et al. Tuberculosis-related deaths within a wellfunctioning DOTS control program. Emerg Infect Dis 2002; 8: 1327-33.

2.- Suárez P G, Watt C J, Alarcón E, Portocarrero J, Zavala D, Canales R, et al. The dynamics of tuberculosis in response to 10 years of intensive control effort in Peru. J Infect Dis 2001; 184: 473-8.

3.- Frieden T R, Sterling T R, Munsiff S S, Watt C J, Dye C. Tuberculosis. Lancet 2003; 362 (9387): 887-99.

4.- Huebner R E, Castro K G. The changing face of tuberculosis. Annu Rev Med 1995; 46: 47-55.

5.- Campos P E, Suárez P G, Sánchez J, Zavala D, Arévalo J, Ticona E, et al. Multidrug-resistant Mycobacterium tuberculosis in HIV-infected persons, Peru. Emerg Infect Dis 2003; 9: 1571-8.

6.- Elliott A M, Halwiindi B, Hayes R J, Luo N, Mwinga A G, Tembo G, et al. The impact of human immunodeficiency virus on mortality of patients treated for tuberculosis in a cohort study in Zambia. Trans R Soc Trop Med Hyg 1995; 89: 78-82.

7.- Dheda K, Lampe F C, Johnson M A, Lipman M C. Outcome of HIV-associated tuberculosis in the era of highly active antiretroviral therapy. J Infect Dis 2004;
190: 1670-6.

8.- Gálvez Hermoso C, Blanco Quintana F, del Amo Valero J, Diez Ruiz-Navarro M, Soriano Vázquez V, González Lahoz J. Effect of antiretroviral treatment and prophylaxis for opportunistic infections on survival of patients with AIDS. Rev Clin Esp 2000; 200: 187-92.

9.- de Jong B C, Israelski D M, Corbett E L, Small P M. Clinical management of tuberculosis in the context of HIV infection. Ann Rev Med 2004; 55: 283-301.

10.- Havlir D V, Barnes PF. Tuberculosis in patients with human immunodeficiency virus infection. N Engl J Med 1999; 340: 367-73.

11.- Lama J R. Epidemiología, clínica y pronóstico de la tuberculosis en los pacientes infectados por el virus de inmunodeficiencia humana. Lima. Perú. Universidad Peruana Cayetano Heredia; 1994.

12.- Mukadi Y D, Maher D, Harries A. Tuberculosis case fatality rates in high HIV prevalence populations in sub-Saharan Africa. AIDS 2001; 15: 143-52.

13.- Thompson B C. Survival rates in pulmonary tuberculosis. Br Med J 1943; 2: 721.

14.- Zachariah R, Spielmann M P, Harries A D, Salaniponi F M. Moderate to severe malnutrition in patients with tuberculosis is a risk factor associated with early death. Trans R Soc Trop Med Hyg 2002; 96: 291-4.

15.- Van Der Sande M A, Schimm van Der Loeff M F, Aveika A A, Sabally S, Togun T, Sarge-
Njie R, et al. Body mass index at time of HIV diagnosis: A strong and independent predictor of survival. J Acquir Immune Defic Syndr 2004; 37: 1288-94.

16. - Tverdal A. Body mass index and incidence of tuberculosis. Eur J Respir Dis 1986; 69: 355-62.

17. - Van Lettow M, Kumwenda J J, Harries A D, Whallen C C, Taha T E, Kumwenda M, et al. Malnutrition and the severity of lung disease in adults with pulmonary tuberculosis in Malawi. Int J Tuberc Lung Dis 2004; 8: 211-7.

18. - Palmero D J, Ambroggi M, Brea A, De Lucas M, Fulgenzi A, Martínez D, et al. Treatment and follow-up of HIV-negative multidrug-resistant tuberculosis patients in an infectious diseases reference hospital, Buenos Aires, Argentina. Int J Tuberc Lung Dis 2004; 8: 778-84.

19.- Bernabé A, Vargas D. Evaluación de un programa controlado de tuberculosis en un centro periférico de Lima. Rev Med Hered 2005; 16: 218-23.

20.- Diagnostic Standards and Classification of Tuberculosis in Adults and Children. This official statement of the American Thoracic Society and the Centers for Disease Control and Prevention was adopted by the ATS Board of Directors, July 1999. This statement was endorsed by the Council of the Infectious Disease Society of America, September 1999. Am J Respir Crit Care Med 2000; 161 (4 Pt 1): 1376-95. 